\title{
Brugada Syndrome Ventricular Arrhythmia by ECG Finding
}

National Cancer Institute

\section{Source}

National Cancer Institute. Brugada Syndrome Ventricular Arrhythmia by ECG Finding. NCI

Thesaurus. Code C71059.

An electrocardiographic finding of a pattern of right bundle branch block and ST-segment elevation within electrocardiogram leads V1-V3. This pattern emerges as a result of a defect in ion channel genes, resulting in atypical electrophysiological activity in the right ventricle and a propensity for malignant tachyarrhythmias. 